\title{
Varióveis fisiológicas \\ cardiovasculares \\ acompanhadas durante \\ o repouso e atividade física: \\ sugestão de um protocolo experimental
}

Diogo Correa Maldonado

Graduado em Fisioterapia - Uninove.

fisiomaldonado@ig.com.br, São Paulo [Brasil]

Mórcio Cantuório Ferreira

Graduando em Fisioterapia - Uninove.

fisiocantuario@ig.com.br, São Paulo [Brasil]

Renata Patrícia Pudo Ribeiro

Graduada em Fisioterapia - Uninove.

fisiorenata9@ig.com.br, São Paulo [Brasil]

\section{Adriana Ferreira Grosso}

Mestre em Entomologia - USP;

Professora na graduação - UnIB/Uninove.

adrianafg@uninove.br, São Paulo [Brasil]
Tanto o estado de repouso fisiológico quanto o exercício representam um desafio para os sistemas de controle na manutenção da homeostasia. Em geral, os sistemas do corpo mantêm um estado estável durante as interações com o ambiente externo (como, por exemplo, mudar a posição do corpo, realizar atividade física), apesar de vários parâmetros fisiológicos variarem. Os parâmetros cardiovasculares são índices interessantes para estudar o equilíbrio do organismo e as ações em prol da homeostase. Para facilitar o estudo dos parâmetros cardiovasculares e suas influências, abordados na teoria da disciplina de Fisiologia Humana, elaborou-se um protocolo experimental que pode ser usado como referência durante as aulas práticas de fisiologia cardiovascular na graduação em Fisioterapia. O protocolo foi criado com a intenção de solidificar os conceitos da fisiologia vascular e cardíaca ao mostrar o comportamento das variáveis fisiológicas durante duas situações: o momento de repouso fisiológico e a situação de atividade física. Adicionalmente, este trabalho vem destacar a importância do conteúdo da disciplina básica no contexto do curso.

Palavras-chave: Atividade física.

Fisiologia cardiovascular. Fisiologia experimental. 


\section{Introdução}

A homeostasia ou homeostase orgânica é considerada o estado de equilíbrio do ambiente interno do corpo, que é mantido por processos dinâmicos de feedback e regulação. Apesar de o organismo estar a todo momento em interação com o ambiente externo, modificando-se organicamente a partir dessa interação, a necessidade e a importância de retornar ao equilíbrio ou situação de homeostase são fundamentais.

O exercício representa um desafio para os sistemas de controle do corpo na manutenção da homeostasia. Em geral, os muitos sistemas de controle do corpo mantêm um estado estável durante a maioria dos tipos de exercício, apesar de vários parâmetros fisiológicos variarem (FIELDING; BEAN, 2001). Segundo Powers e Howley (2000), uma excelente ilustração do controle homeostático utilizado é o sistema dos baroceptores, responsável pela regulação da pressão arterial (PA). Os baroceptores, localizados nas artérias carótidas e no arco aórtico, são receptores sensíveis à PA: quando a PA aumenta além dos níveis normais, eles são estimulados e impulsos nervosos transmitidos ao centro de controle localizado no bulbo; este centro, por sua vez, diminui o número de impulsos transmitidos ao coração, reduzindo a quantidade de sangue bombeado por ele e fazendo com que a pressão volte ao normal (NEDER; NERY, 2003).

Além do controle pelo sistema dos baroceptores e pela atividade neural, há o ajuste da PA por meio da adequação da volemia pelo sistema da renina-angiotensinaaldosterona (GUYTON; HALL, 2002). Esse sistema é requerido pelo organismo quando há alteração lenta da PA, necessitando das ações renais e endócrinas para adequação do volume dos líquidos corporais.
A PA é determinada diretamente pelo volume sangüíneo e pela complacência arterial. Tais determinações são influenciadas pelo débito cardíaco (DC), pelo débito sistólico (DS), pela freqüência cardíaca (FC), pela freqüência respiratória (FR) e pela resistência periférica total (RPT). Situações como a posição do corpo do indivíduo, a atividade e as variações de temperatura são fatores modificadores da PA e, por conseguinte, dos parâmetros ligados a ela. Esses parâmetros são conhecidos como fatores hemodinâmicos.

Dessa forma, o sistema circulatório é dotado de mecanismos sistêmicos e regionais para o controle da PA, variando as condições dos parâmetros vasculares e cardíacos para adequar o organismo às mudanças de situação. Dentro desses parâmetros está o DS, que é avaliado no momento de ejeção cardíaca, enquanto o volume diastólico corresponde ao relaxamento muscular cardíaco para receber fluxo sangüíneo. Ambos os volumes são encontrados ao se aferir a PA e dependem do retorno venoso (RV).

Os ajustes cardiovasculares requerem, em qualquer situação, uma adaptação - de acordo com a oferta e o consumo de oxigênio no organismo - conseguida por meio da respiração (NEDER; NERY, 2003).

A importância do controle da pressão é que ele impede que as alterações do fluxo sangüíneo por uma área do corpo possam alterar, de forma significativa, o fluxo em algum outro ponto do corpo (GUYTON; HALL, 2002).

Em fisiologia cardiovascular, as variáveis que influenciam e que determinam a PA de um indivíduo sob diferentes condições (mudanças de posição do corpo, variações térmicas, exigências metabólicas e outras) são apresentadas e estudadas na teoria e geram dificuldades quanto ao entendimento do 
dinamismo dos controles. A prática que demonstra a ocorrência freqüente de situações modificadoras da homeostase cardiovascular, que influencia especialmente os parâmetros de PA e a existência dos mecanismos reguladores de feedback, não só fortalece os conceitos teóricos, mas também torna o tema mais acessível à compreensão pelo alunado.

Assim, a finalidade deste trabalho foi elaborar um protocolo experimental que possa ser usado como referência durante as aulas práticas de fisiologia cardiovascular e fisiopatologia na graduação em Fisioterapia.

Este protocolo deve solidificar os conceitos teóricos da fisiologia vascular e cardíaca ao mostrar o comportamento das variáveis fisiológicas durante duas situações comparáveis: o momento de repouso fisiológico e a situação de atividade física.

\section{Materiais e método}

Este trabalho foi desenvolvido nos laboratórios de Fisiologia e Clínica de Fisioterapia do Centro Universitário Nove de Julho (Uninove), nos campi Memorial e Vila Maria, em 2003 e 2004. Contou com uma pesquisa entre os professores de Fisioterapia sobre quais seriam as principais dificuldades dos alunos na aplicação dos conceitos de fisiologia cardiovascular. Em seguida, procurou-se por procedimentos experimentais na literatura (CABRERA; ROSA; PERALTA, 1998; RUSSELL, 1978; THARP; WOODMAN, 2002) e também se realizou a análise dos experimentos freqüentemente aplicados na disciplina (GROSSO, 2000). Os experimentos selecionados para teste tinham de demonstrar claramente os parâmetros fisiológicos em questão e deveriam utilizar equipamentos e/ou técnicas que fossem de conhecimento ou utilização do profissional de Fisioterapia (WASSERMAN et al., 1999; WEBER et al., 1988).

\section{Resultados e discussão}

O protocolo experimental elaborado pela equipe propõe o acompanhamento de variáveis fisiológicas durante uma situação de repouso fisiológico e durante atividade física em esteira.

Cada protocolo consta de recomendações iniciais, objetivos, materiais e procedimentos.

\subsection{Protocolo para esfigmomanometria e as influências fisiológicas}

Algumas recomendações importantes para se proceder corretamente à verificação da PA. Aferir a pressão sangüínea é um procedimento simples, mas que merece alguns cuidados para assegurar a leitura correta:

- É comum realizá-la mais de uma vez para tirar uma média e obter um valor mais realista;

- Se a pressão alta for detectada na primeira avaliação, normalmente se fazem outras leituras em outros dias para confirmar o valor;

- Os valores de PA obtidos, especialmente quando elevados, implicam novas medições periódicas para controle;

- Aparelho devidamente calibrado e manguito adequado ao diâmetro do braço do voluntário ou paciente - há vários modelos no mercado;

- Antes de iniciar os procedimentos ou quando realizá-los mais de uma vez, observar entre eles breve repouso do voluntário ou paciente; 
- Durante a verificação, manter o braço do voluntário ou paciente à altura do átrio esquerdo;

- Evitar o acoplamento do aparelho de verificação de pressão sobre blusa ou casaco, pois isso dificulta a captação da pulsação e a ausculta;

- Recomendar ao voluntário que evite fumo, ingestão de álcool, café e medicamentos que possam interferir nos resultados.

\subsubsection{Objetivos do experimento}

Aprender a seqüência de procedimentos para aferir a PA.

Acompanhar a influência de fatores fisiológicos sobre a PA de um indivíduo.

\subsubsection{Material}

Algodão e álcool;

Esfigmomanômetro;

Estetoscópio;

Fita métrica;

Maca e cadeira;

Recipiente contendo gelo.

\subsubsection{Procedimentos}

Certificar-se de que o estetoscópio e o esfigmomanômetro estejam íntegros e o esfigmomanômetro calibrado e desinsuflado antes de ser ajustado ao membro do voluntário - segundo American Heart Association (apud JESUS; JESUS, 2000?).

\subsubsection{Posição de decúbito}

Posicionar o voluntário deitado em uma maca, em local calmo e confortável, com o braço esquerdo apoiado ao nível do coração, permitindo cinco minutos de repouso. Esclarecer ao voluntário os procedimentos a que será submetido, a fim de diminuir a ansiedade. Em seguida, descobrir o membro a ser aferido e medir a circunferência do braço para assegurar-se do tamanho do manguito. Selecionar o tamanho ideal do manguito a ser utilizado, o qual deve corresponder a $40 \%$ da circunferência braquial em relação à largura e $80 \%$ para o comprimento.

Medir a distância entre o acrômio e o olecrano, colocando o manguito no ponto médio.

Envolver, com o manguito, o braço do voluntário, mantendo o aparelho a dois centímetros de distância da sua margem inferior à fossa antecubital, posicionando o centro do manguito sobre a artéria braquial, permitindo que tubos e conectores estejam livres e o manômetro em posição visível para o experimentador.

Palpar a artéria braquial e centralizar o manguito.

Com a mão não dominante, palpar a artéria radial (utilizar os dedos indicador e médio) e, simultaneamente, com a mão dominante, fechar a saída de ar (válvula da pêra do esfigmomanômetro), inflando rapidamente a bolsa até 70 milímetros de mercúrio $(\mathrm{mmHg})$ e aumentando, gradualmente, a pressão aplicada até que se perceba o desaparecimento do pulso, inflando $10 \mathrm{mmHg}$ acima deste nível. Desinsuflar o manguito lentamente, identificando, pelo método palpatório, a pressão arterial sistólica.

É importante repetir os procedimentos pelo menos duas vezes, para que se tenha certeza do resultado; no entanto, deve-se aguardar de 15 a 30 segundos para inflar novamente o manguito, a fim de que o voluntário regularize sua pressão.

Até esse momento, foi verificado o valor máximo de ajuste do manômetro para o voluntário sob procedimento. A PA ainda não foi aferida. Posicionar corretamente as olivas do estetoscópio no canal auricular do expe- 
rimentador, certificando-se da ausculta adequada na campânula (a posição correta das olivas do estetoscópio é para frente em relação ao diafragma, pois permite maior adequação ao conduto auricular, diminuindo a interferência de ruídos ambientais externos).

Com a mão não dominante, posicionar a campânula do estetoscópio sobre a artéria braquial, palpada abaixo do manguito na fossa antecubital; simultaneamente, com a mão dominante, fechar a saída de ar (válvula da pêra do esfigmomanômetro) e, em segui$\mathrm{da}$, insuflar o manguito gradualmente até o valor da pressão arterial sistólica estimada pelo método palpatório. Continuar insuflando rapidamente até $20 \mathrm{mmHg}$ acima desta pressão. Desinsuflar o manguito de modo que a pressão caia de 2 a $4 \mathrm{mmHg}$ por segundo. Identificar, pelo método auscultatório, a pressão sistólica ou pressão máxima (em $\mathrm{mmHg}$ ), observando no manômetro o ponto correspondente ao primeiro ruído regular audível (sons de Korotkoff), a pressão diastólica ou pressão mínima (em $\mathrm{mmHg}$ ) e o ponto correspondente à cessação dos ruídos de Korotkoff. Desinsuflar totalmente o manguito.

Colocar o voluntário para descansar por cinco minutos, antes de repetir todo o procedimento. Registrar a posição em que o voluntário se encontrava no momento da verificação da PA, o tamanho do manguito e do membro utilizados e os valores da PA (em $\mathrm{mmHg}$ ).

\subsubsection{Posição ortostática}

Repetir os procedimentos anteriores, mas agora com o voluntário em pé. Ao final, registrar a posição em que o voluntário se encontrava no momento da verificação da PA, o tamanho do manguito e do membro utilizados e os valores da PA (em $\mathrm{mmHg}$ ).

\subsubsection{Sentado e sob influência de temperatura reduzida}

Repetir os procedimentos anteriores, mas agora com o voluntário sentado, anotando os valores de pressão sistólica e diastólica.

Solicitar ao voluntário que mergulhe sua mão direita em um recipiente (à temperatura ambiente de $5^{\circ} \mathrm{C}$ ) contendo gelo, assim permanecendo por um minuto. Em seguida, aferir a pressão e anotar os valores.

O esfigmomanômetro deve estar ajustado ao braço esquerdo e o braço direito em contato com o gelo.

\subsection{Protocolo para parâmetros cardiovasculares na atividade física}

Algumas recomendações baseadas em Barros Neto, César e Tambeiro (1999) necessárias a esta atividade:

- Solicitar ao voluntário que venha com roupa adequada à atividade física (short e tênis apropriados para caminhada);

- Questionar quanto ao uso de medicamentos, tabagismo, conhecimento de doenças cardiovasculares e/ou respiratórias preexistentes, labirintite, dores musculares, incapacidades musculoesqueléticas como osteoartrose e descoordenação motora à corrida. Para realizar esta atividade, o voluntário deve estar em jejum. Convém descartar a participação do voluntário que apresenta doenças cardiorespiratórias, labirintite, dores de qualquer natureza e incapacidades músculoesqueléticas. É importante realizar alongamento dos membros inferiores, antes de iniciar a atividade física na esteira, alongando 
principalmente a região posterior da coxa;

- Anotar idade, sexo e as observações referentes ao item 2 e utilizar protocolo apresentado ao final da descrição do experimento (Quadro 1);

\begin{tabular}{|c|c|c|c|c|c|}
\hline \multicolumn{6}{|l|}{ Nome } \\
\hline \multicolumn{2}{|l|}{ Idade } & \multicolumn{4}{|l|}{ Sexo } \\
\hline \multicolumn{6}{|l|}{ Data } \\
\hline \multicolumn{6}{|l|}{ Observações } \\
\hline \multirow[t]{2}{*}{ Estágio } & \multirow{2}{*}{$\begin{array}{c}\text { Pressão } \\
\text { arterial } \\
\text { (PA) }\end{array}$} & \multirow{2}{*}{$\begin{array}{c}\text { Função } \\
\text { cardíaca } \\
\text { (FC) }\end{array}$} & \multirow{2}{*}{$\begin{array}{l}\text { Freqüência } \\
\text { respiratória } \\
\text { (FR) }\end{array}$} & \multicolumn{2}{|c|}{$\begin{array}{c}\text { Escala de } \\
\text { esforço }\end{array}$} \\
\hline & & & & M & $\mathrm{R}$ \\
\hline \multicolumn{6}{|l|}{$\begin{array}{c}\text { Em repouso } \\
\text { inicial }\end{array}$} \\
\hline \multicolumn{6}{|l|}{$\begin{array}{l}\text { Após cinco } \\
\text { minutos em } \\
\text { velocidade } \\
4,5 \mathrm{Km} / \mathrm{h}\end{array}$} \\
\hline \multicolumn{6}{|l|}{$\begin{array}{l}\text { Após cinco } \\
\text { minutos em } \\
\text { velocidade } \\
5,5 \mathrm{Km} / \mathrm{h}\end{array}$} \\
\hline $\begin{array}{c}\text { Após } \\
\text { repouso final }\end{array}$ & & & & & \\
\hline
\end{tabular}

Quadro 1: Quadro para anotação das condições e respostas cardiovasculares durante $\alpha$ atividade física

Obs.: Incômodo de origem muscular (M) ou respiratória (R).

Fonte: Os autores.

- Questionar o voluntário sobre suas condições físicas gerais, utilizando, para isso, a escala de esforço. A intenção é tomar conhecimento de qualquer desconforto do voluntário que o leve a alterações repentinas do estado fisiológico, principalmente no que se refere a incômodos de origem muscular (M) e/ou respiratória (R). A escala de esforço (Quadro 2) é dada em números e deve ser mostrada ao voluntário durante a sua atividade na esteira;

\begin{tabular}{|c|c|}
\hline Valor & Significado \\
\hline 1 & Nenhum \\
\hline 2 & Muito, muito pouco \\
\hline 3 & Muito pouco \\
\hline 4 & Pouco \\
\hline 5 & Moderado \\
\hline 6 & Pouco intenso \\
\hline 7 & Intenso \\
\hline 8 & Muito intenso \\
\hline 9 & Muito, muito intenso \\
\hline 10 & Máximo \\
\hline
\end{tabular}

Quadro 2: Escala de esforço para avaliação dass condições cardiorespiratórias do voluntório durante $\alpha$ atividade física

Fonte: Os autores.

- Cada número representa uma condição ou desconforto respectivo. O voluntário irá, então, relacionar o número à sua situação durante a atividade física.

Sugestão: realizar este experimento com voluntários sedentários em treinamentos, a fim de que se possam comparar os resultados.

\subsubsection{Objetivos do experimento}

Treinar a seqüência de procedimentos para aferir a PA.

Acompanhar a influência de fatores fisiológicos sobre a PA de um indivíduo, durante uma situação de exercício físico.

\subsubsection{Material}

Cronômetro;

Escala de esforço;

Esfigmomanômetro;

Esteira elétrica;

Estetoscópio;

Freqüencímetro com relógio;

Maca;

Protocolo para anotações. 


\subsubsection{Procedimentos}

Inicialmente, para facilitar a passagem de corrente elétrica, deve ser feita a umidificação do freqüencímetro, posicionando-o em contato direto com a pele do voluntário (não pode estar sobre a roupa) e, em seguida, ajustá-lo à altura dos mamilos. $\mathrm{O}$ voluntário também deve colocar o relógio do freqüencímetro.

Depois, ajustar o esfigmomanômetro na posição correta, ou seja, no membro superior esquerdo do voluntário.

Com o voluntário em repouso, deitado ou sentado, os experimentadores devem aferir a PA (como descrito em experimento anterior), verificar a FC por meio da leitura do freqüencímetro e fazer a contagem da FR pela observação da expansão torácica (movimento de inspiração + movimento de expiração = uma expansão), anotando os valores correspondentes.

Pedir ao voluntário que se posicione na esteira elétrica e ligue o equipamento em $0 \%$ de inclinação e com velocidade de 4,5 quilômetros por hora $(\mathrm{km} / \mathrm{h})$, exercitando-se, nesse ritmo, por cinco minutos. Na seqüência, solicitar que permaneça em atividade na esteira, mesmo depois de terminado o tempo proposto. Os experimentadores devem aferir novamente a PA (com o voluntário em movimento), verificando as FC e FR e anotando todos os valores (Fotografia 1).

A partir do sétimo minuto, a velocidade da esteira deve ser aumentada para $5,5 \mathrm{~km} / \mathrm{h}$, mantendo $0 \%$ de inclinação, com caminhada por cinco minutos. Ao final, e ainda com o voluntário em movimento, avaliar mais uma vez a PA e as FC e FR. Os experimentadores devem fazer o registro dos valores encontrados.

Posteriormente, desacelera-se a esteira para $3 \mathrm{~km} / \mathrm{h}$ e, nos próximos dois minutos, para $1 \mathrm{~km} / \mathrm{h}$ até que pare por completo, evitando-se as interrupções abruptas para não causar hipotensão no voluntário. Após um período de dois minutos em repouso, a PA, as FC e FR do voluntário devem ser novamente verificadas e anotadas pelos experimentadores.

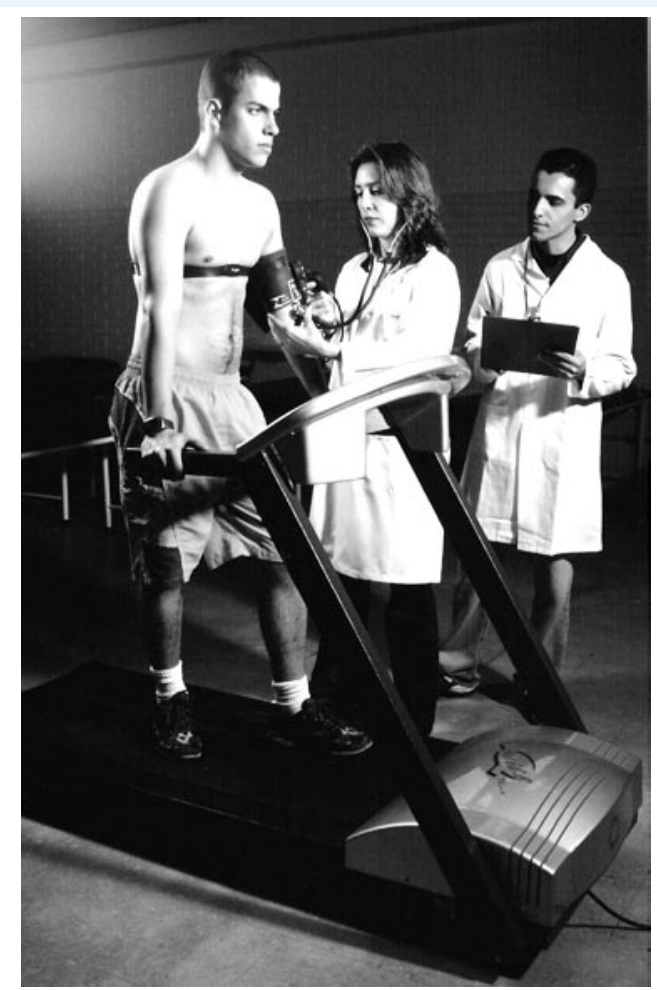

Fotografia 1 - Posicionamento do voluntário na esteira durante a atividade física e avaliação pelos experimentadores Fonte: Os autores.

\section{Conclusão}

A execução do protocolo experimental proposto chama a atenção para o comportamento das variáveis fisiológicas cardiovasculares durante o repouso e durante a atividade física. Baseado nos parâmetros determinantes da homeostase metabólica, durante o repouso ou na execução de exercício controlado, foi realizada a construção deste 
protocolo experimental, enfocando as variáveis cardiovasculares passíveis de ser acompanhadas em ambas as situações: PA, FC e FR e propõem-se discussões acerca da fisiologia cardiovascular.

Pela prática laboratorial, o estudante terá a oportunidade de vivenciar situações que se assemelham ou que até representam as situações diárias que afetam um organismo e de perceber que variáveis fisiológicas, tais como as cardiovasculares, respondem a esses diferentes momentos para se ajustarem à homeostase.

O aprendizado mais consistente e de longo prazo solidifica-se com a percepção do aluno de que o conteúdo teórico é passível de visualização e comprovação experimental. Tal consistência vem com a proposta de questionamento e discussões, pela análise dos dados obtidos na experimentação.

A realização de experimentos sempre facilita e complementa a compreensão da teoria, além de mostrar a aplicabilidade da disciplina no contexto do curso.

\section{Cardiovascular physiological variables examined during the rest state and the exercise activity: suggestion of on experimental protocol}

Both the physiological rest state and the exercise activity represent a challenge for the control systems of the homeostasy maintenance. In general, the body systems maintain a stable state during the interactions with the external environment (as, for instance, while changing the body position or doing physical activities), despite the variation of many physiological parameters. The cardiovascular parameters are interesting indexes to study the organism equilibrium and the actions that yield homeostasis. In order to make easier the understanding of the cardiovascular parameters and their influences during the classes of the discipline of Human Physiology, an experimental protocol was elaborated to be used as reference during the practical classes of cardiovascular physiology during the graduation course on Physiotherapy. The protocol aims to improve the theoretical concepts of vascular and cardiac physiology showing the behavior of physiological variables during two situations: at rest and during physical activities. Additionally, this work shows up the importance of this basic discipline subject in the course context.

Key words: Cardiovascular physiology. Experimental physiology. Physical activities.

\section{Referências}

BARROS NETO, T. L. de; CÉSAR, M. de C.; TAMBEIRO, V. L. Avaliação da aptidão física cardiorrespiratória. In: GHORAYEB, N.; BARROS NETO, T. L. de (Org.). O exercício: preparação fisiológica, avaliação médica, aspectos especiais e preventivos. 1. ed. São Paulo: Atheneu, 1999. p. 15-24.

CABRERA, M. A. de; ROSA, R. A. C.; PERALTA, C. C. Fisiologia: aprendendo no laboratório. 1. ed. São Paulo: Sarvier, 1998.

FIELDING, R. A.; BEAN, J. Adaptações fisiológicas ao exercício dinâmico. In: FRONTERA, W. R.; DAWSON, D. M.; SLOVIK, D. M. Exercício físico e reabilitação. 1. ed. Porto Alegre: Artmed, 2001. p. 59-84.

GROSSO, A. F. (Org.). Aulas práticas de fisiologia. São Paulo: Uninove, 2000. (mimeo).

GUYTON, A. C.; HALL, J. E. Tratado de fisiologia médica. 10. ed. Rio de Janeiro: Guanabara Koogan, 2002. 
JESUS, P. C. de; JESUS, C. A. C. de. Técnica da medida indireta da pressão arterial. Brasília, DF: UnB, [2000?]. Disponível em: <http://www.unb.br/fs/enf/nipe/ tecnicapa.html>. Acesso em: 14 mar. 2004.

NEDER, J. A.; NERY, L. E. Fisiologia clínica do exercício: teoria e prática. 1. ed. São Paulo: Artes Médicas, 2003.

POWERS, S. K.; HOWLEY, E. T. Testes de esforço físico para a avaliação da função cardiorrespiratória. In: . Fisiologia do exercício: teoria e aplicação ao condicionamento e ao desempenho. 3. ed. São Paulo: Manole, 2000. p. 265-278.
RUSSELL, G. K. Laboratory investigations in human physiology. New York: Macmillan, 1978.

THARP, G. D.; WOODMAN, D. A. Experiments in physiology. 8. ed. San Francisco: Benjamin Cummings, 2002.

WASSERMAN, K. et al. Principles of exercise testing and interpretation: including pathophysiology and clinical applications hardbound. 3. ed. Philadelphia: Lippincott Williams \& Wilkins, 1999.

WEBER, K. T. et al. Concepts and applications of cardiopulmonary exercise testing. Chest, Northbrook, v. 93, p. 843-847, 1988.

\section{Para referenciar este texto:}

MALDONADO, D. C. et al. Variáveis fisiológicas cardiovasculares acompanhadas durante o repouso e atividade física: sugestão de um protocolo experimental. ConScientiae Saúde, São Paulo, v. 4, p. 33-41, 2005. 
